PVP2013-97426

\title{
EVOLUTION OF MECHANICAL BEHAVIOR OF 6XXX ALUMINIUM ALLOY DUE TO THE PRECIPITATION STATE DURING A THERMO-MECHANICAL PROCESS
}

\author{
Didier Bardel \\ LaMCoS UMR CNRS 5259 \\ INSA-Lyon \\ Université de Lyon \\ F69621 Villeurbanne, France \\ Thibaut Chaise \\ LaMCoS UMR CNRS 5259 \\ INSA-Lyon \\ Université de Lyon \\ F69621 Villeurbanne, France
}

\author{
Michel Perez \\ MATEIS UMR CNRS 5510 \\ INSA-Lyon \\ Université de Lyon \\ F69621 Villeurbanne, France
}

\author{
Jerôme Garnier \\ DEN/DMN/SRMA \\ CEA Saclay \\ Gif sur Yvette, France
}

\author{
Daniel Nelias* \\ LaMCoS UMR CNRS 5259 \\ INSA-Lyon \\ Université de Lyon \\ F69621 Villeurbanne, France \\ Email: daniel.nelias@insa-lyon.fr
}

\author{
Florent Bourlier \\ Mechanical Engineering Lyon Department \\ Materials and Chemistry Section (PEELM-F) \\ AREVA NP \\ F-69456 LYON, France
}

\begin{abstract}
The aim of this research is to link the microstructural state and the mechanical properties of an age hardening alloy during a fast heat treatment such as encountered during welding.

A coupled model between precipitation state and mechanical properties is used to predict the yield strength and hardening behavior that can be observed experimentally. The method permits the identification of the kinematic and isotropic contributions in the hardening model. The methodology is applied to a 6061-T6 aluminium alloy which is used in the Jules Horowitz reactor vessel.

The general idea of this methodology is to couple an efficient microstructural model to a mechanical one based on the dislocation theory and ad'hoc experiments. The theoretical background is based on the work of Kampmann and Wagner, known as the KWN model, to account for nucleation, growth/dissolution and coarsening of precipitates. This analysis requires transient thermo-mechanical experimental data. The efficiency of these models and their coupling are shown for a serie 6XXX aluminium alloy which contains $\beta^{\prime \prime}$ and $\beta^{\prime}$ precipitates. Ultimately these
\end{abstract}

*Address all correspondence to this author. models are coupled to a FEA model and allows to predict the distribution of precipitates within each element of the mesh, and subsequently its mechanical behavior.

\section{NOMENCLATURE}

$l \quad$ Length of needle.

$D_{M g} \quad$ Diffusion coefficient of magnesium.

$D_{S i} \quad$ Diffusion coefficient of silicon.

$R_{p} \quad$ Tip radius of needle.

$X_{M g}^{0} \quad$ Atomic fraction of magnesium in matrix.

$X_{M g}^{i} \quad$ Atomic fraction of magnesium at matrix/precipitate interface.

$X_{M g}^{p} \quad$ Atomic fraction of magnesium in precipitate.

$X_{S i}^{0} \quad$ Atomic fraction of silicon in matrix.

$X_{S i}^{i} \quad$ Atomic fraction of silicon at matrix/precipitate interface.

$X_{S i}^{p} \quad$ Atomic fraction of silicon in precipitate.

$\gamma \quad$ Matrix/precipitate interface energy.

$K_{s} \quad$ Solubility product of precipitate.

$\xi \quad$ Needle aspect ratio.

$k \quad$ Boltzmann constant. 
$V_{m o l}^{p} \quad$ Molecular volume of precipitate.

$\alpha_{m / p} \quad$ Ratio of atomics volumes for precipitate and matrix.

$\sigma_{y} \quad$ Yield stress.

$\sigma_{0} \quad$ Yield stress of pure aluminium.

$k_{i} \quad$ Element strengthening constant of element ' $\mathrm{i}$ '.

$C_{S S}^{i} \quad$ Element weight content of element 'i'.

$M \quad$ Taylor's factor.

$b \quad$ Burger parameter.

$l_{i} \quad$ Length of precipitate ' $i$ ' in the distribution.

$N_{i} \quad$ Number of precipitates 'i' per volume in the distribution.

$\Gamma \quad$ Tension line of dislocation.

$N_{T O T}$ Total number of simulated precipitates.

\section{INTRODUCTION}

The work presented in this paper is a part of the study of early qualification of pressure vessel for a new French experimental reactor. The approximate size of this piece is five meters high with a diameter about one meter. To obtain this piece made of AA6061-T6, several shells are welded together using a welding process: the electron beam. The ultimate goal is to predict the metallurgical state generated by the welding process and to deduce the mechanical properties and residual stresses in the vessel.

To achieve this goal, this work tries to understand the link between microstructural evolution and mechanical properties. In a first part, the implementation of a microstructural model is presented. This implementation is based on the works of Hillert [1]. This model is validated using measurement of Small Angle Neutron Scattering (SANS) and Transmission Electron Microscopy (TEM). These experimental campaigns provide informations about the distribution of precipitates present in the alloy for several heat treatment representative of welding. For SANS, an ellipsoidal distribution is using to have a good fitting of raw diffusion spectra. The microstructural state of the alloy for rapid heat treatments can then be provided by finite element simulations. In a second part, a modelisation is presented to link the precipitation state and mechanical properties. This modelisation is validated with cyclic tensile tests performed on various precipitation states.

\section{Microstructural modelisation}

The KWN model allows to predict the different microstructural phenomena that can occur during various heating kinetics encountered in a welding process: nucleation, growth, and coarsening. This model is generally implemented for spherical precipitates ( [2] [3] ) but for the aluminium alloys this hypothesis is not acceptable because precipitates have often large aspect ratio. Indeed, for the 6000 series the Heat Affected Zone (HAZ) is composed by needle shaped precipitates $\left(\beta^{\prime \prime}\right.$ and $\left.\beta^{\prime}\right)$ [4].

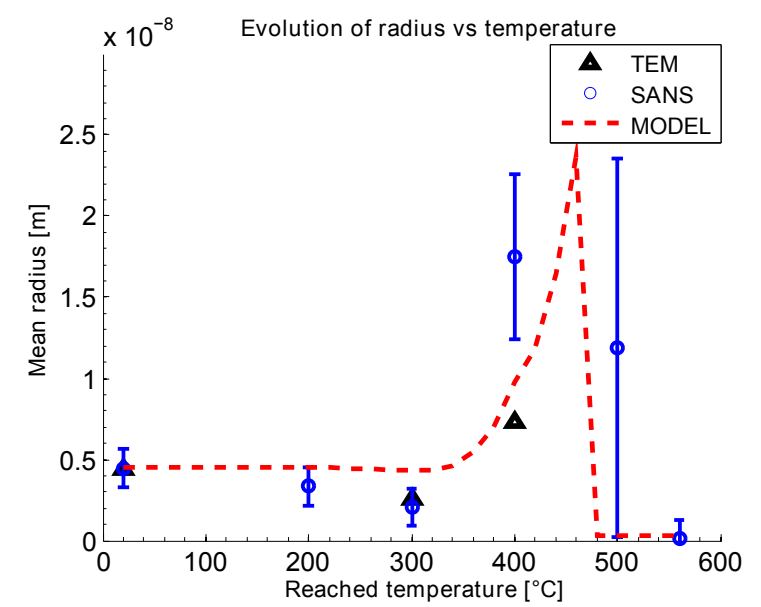

FIGURE 1: SIMULATED VS EXPERIMENTAL MEAN RADIUS OF NEEDLES.

Here, Classical Nucleation and Growth Theories (CNGTs) have been adapted to model accurately the precipitation of needle shaped particles. Hillert proposed a modification of the Zener growth equation considering a constant tip radius but without coarsening treatment. Here, an adaptation of this work is presented to have a diffusion/coarsening stage. This adapted expression is implemented in a 'Lagrangian-like' model class management software (PreciSo) to obtain the distribution density of precipitates. Thus, the evolution of precipitate length can be written as follows for a constant aspect ratio [5]:

$$
\left\{\begin{array}{l}
\frac{d l}{d t}=\frac{3 D_{M g}}{4 R_{p}} \frac{X_{M g}^{0}-X_{M g}^{i}}{\alpha_{m / p} X_{M g}^{p}-X_{M g}^{i}} \\
\frac{d l}{d t}=\frac{3 D_{S i}}{4 R_{p}} \frac{X_{S i}^{0}-X_{S i}^{i}}{\alpha_{m / p} X_{S i}^{p}-X_{S i}^{i}} \\
X_{M g}^{i}{ }^{x} X_{S i}^{i}{ }^{y}=K_{s} \cdot \exp \left(\frac{\xi}{\xi-2 / 3} \frac{4 V_{m o l}^{p} \gamma}{3 R k T}\right)
\end{array}\right.
$$

Here, as in [2] a fictitious phase is proposed with $\beta^{\prime \prime}-\beta^{\prime}$ nature. This equivalent phase allows to obtain a good representation of the microstructural state for several thermal loads representative of welding. These simulations are compared with both TEM and SANS measurements applied on tensil test samples [5].

\section{Mechanical tests}

Tensile tests have been carried out at room temperature on specimens previously heated up to peak temperatures of 473 , $573,673,773$ and $833 \mathrm{~K}$ and with various heating rates $(0.5,5$, $15,50,200 \mathrm{~K} / \mathrm{s}$ ) in order to measure their mechanical properties. The thermal loadings are representative of the thermal histories encountered in Electron Beam welding [6]. The experimental 


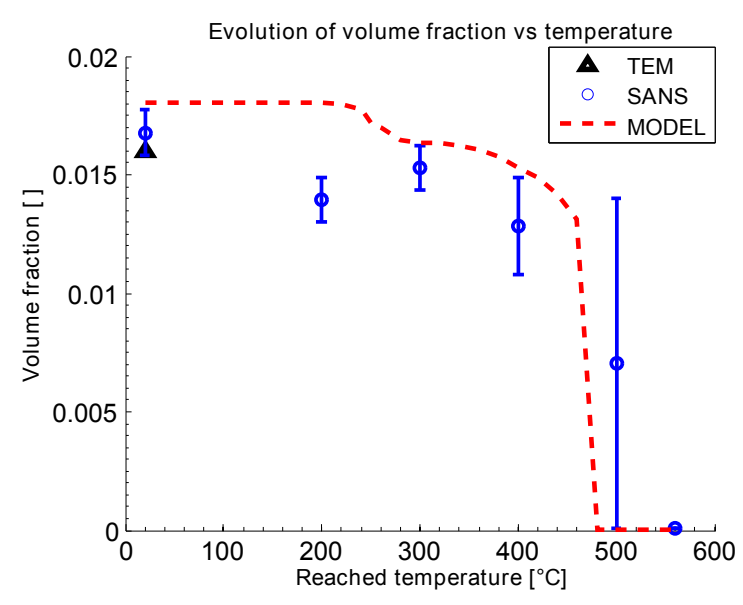

FIGURE 2: SIMULATED VS EXPERIMENTAL VOLUME FRACTION OF NEEDLES.

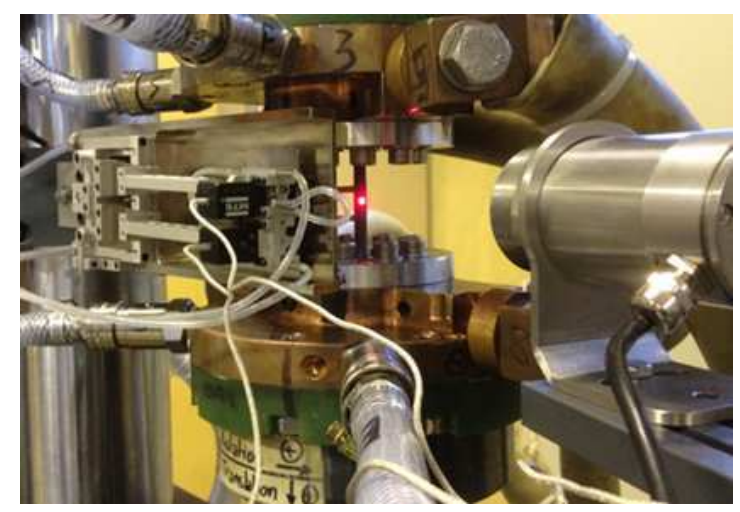

\section{FIGURE 3: INSTRUMENTATION FOR TENSILE TESTS.}

device that is used here has been specifically developed at INSA Lyon to meet these kind of requirements [7].

The precipitation state is then used as an input parameter of a mean field micro-mechanical models that predict the yield strength of the alloy.

$$
\sigma_{y}=\sigma_{0}+\sum k^{i} \cdot C_{S S}^{i}{ }^{2 / 3}+\frac{M}{b} \sqrt{\frac{\sum N_{i} l_{i}}{2 \sqrt{3} \Gamma}}\left(\frac{\sum_{i} N_{i} F_{i}}{N_{T O T}}\right)^{3 / 2}
$$

These results are successfully compared to tensile tests experiments performed on various heating conditions, representing the heat-affected zone of a weld. In this paper the influence of several assumptions on the yield stress modeling have been reviewed and new equations are used [5]. The use of a full distribution approach allows to improve the physical meaning of the

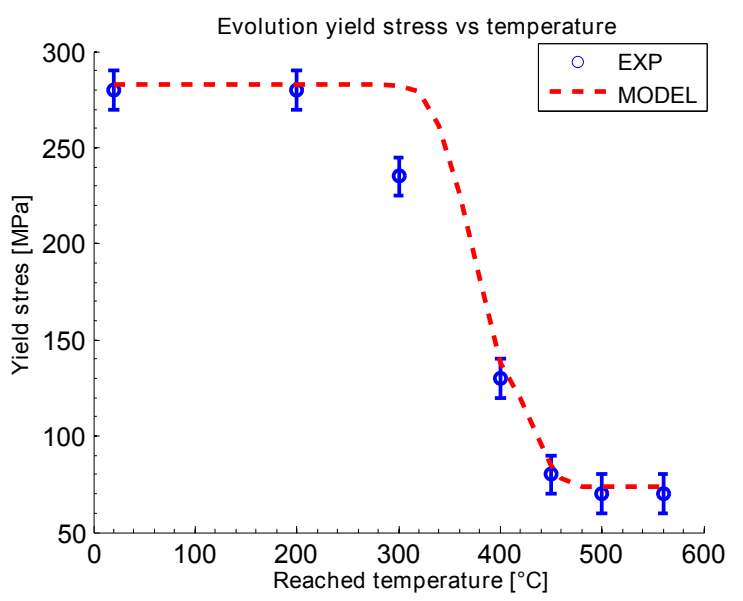

FIGURE 4: YIELD STRESS PREDICTION VS EXPERIMENTS.

fitted parameters. Here, only one fitted parameter is used and the identified Taylor's factor is perfectly compatible with recent works on aluminium alloy [8].

Then, for hardening curves, classical elastoplastics laws can be used such as Amstrong-Frederic models [9]. In these laws, the yield stress is predicted by the previous predicted approch. Other coefficients values are dependant on the simulated precipitates mean radius and volume fraction.

\section{CONCLUSION AND PERSPECTIVES}

In this paper, a coupled physical precipitation/yield stress modelisation is presented. In the first part, an adjustment strategy is proposed for a single fictitious phase to fit microstructural evolution obtained by SANS. Then, yield stress prediction are validated with tensile tests. This modelisation provides a good fitting in a wide range of thermals histories and can be used in finite element software with phenomenological plasticty models such as Amstrong Frederic's.

The prospect of this work is the identification of viscous part of mechanical behaviour. Then, all the tools will be available for prediction of residual stresses for age hardening aluminium alloys.

\section{ACKNOWLEDGMENT}

The authors gratefully acknowledge Areva and the French Atomic Energy Commission (CEA) for industrial and financial support. The staff of the D22 beamline of Laue Langevin Institut is thanked for technical help and Alexis Deschamps for his SANS advices. 


\section{REFERENCES}

[1] Hillert, M., Hoglund, L., and Agren, J., 2003. "Diffusioncontrolled lengthening of widmansttten plates". Acta Materialia, 51, pp. 2089-2095.

[2] Myhr, O. R., Grong, O., and Andersen, S. J., 2001. "Modelling of the age hardening behaviour of al-mg-si alloys". Acta Materialia, 49, pp. 65-75.

[3] Simar, A., Bréchet, Y., de Meester, B., Denquin, A., and Pardoen, T., 2007. "Sequential modeling of local precipitation, strength and strain hardening in friction stir welds of an aluminum alloy 6005a-t6". Acta Materialia, 55, pp. 61336143.

[4] Myhr, O., Grong, O., Fjaer, H., and Marioara., C., 2004. "Modelling of the microstructure and strength evolution in al-mg-si alloys during multistage thermal processing”. Acta Materialia, 52, pp. 4997-5008.

[5] Bardel, D., Perez, M., Nelias, D., Maisonnette, D., Hutchinson, C., Deschamps, A., and Chaise, T., 2013. "Evolution of precipitation state and strength for welding of a 6061 aluminium alloy". Submitted.

[6] Maisonnette, D., 2010. "Influences mcaniques et mtallurgiques de procds haute temprature sur un alliage daluminium 6061-t6". PhD thesis, Insa Lyon.

[7] Maisonnette, D., Suery, M., Nelias, D., Chaudet, P., and Epicier, T., 2011. "Effects of heat treatments on the microstructure and mechanical properties of a 6061 aluminium alloy". Materials Science and Engineering A, 528, pp. 2718-2724.

[8] Starink, M., and Wang, S., 2003. "A model for the yield strenght of overaged al-zn-mg-cu alloys". Acta Materialia, 51, pp. 5131-5150.

[9] Jiang, Y., and Kurath, P., 1996. "Characteristics of the armstrong-frederick type plasticity models". International Journal of Plasticity, 12, pp. 387-415. 